\title{
Younger but not older adults benefit from salient feedback during learning
}

\author{
Michael Herbert ${ }^{1}$, Ben Eppinger ${ }^{2}$ and Jutta Kray ${ }^{*}$ \\ Development of Language, Learning, and Action, Department of Psychology, Saarland University, Saarbruecken, Saarland, Germany \\ 2 Max Planck Institute for Human Development, Berlin, Germany
}

Edited by:

Shulan Hsieh, National Cheng Kung

University, Taiwan

Reviewed by:

Marco Steinhauser, University of

Konstanz, Germany

Stephen B. R. E. Brown, Leiden

University, Netherlands

${ }^{*}$ Correspondence:

Jutta Kray, Development of Language,

Learning, and Action, Department of

Psychology, Saarland University, P.O.

Box 1511 50, D-66041 Saarbruecken,

Germany.

e-mail:j.kray@mx.uni-saarland.de
Older adults are impaired in reinforcement learning $(R L)$ when feedback is partially ambiguous (e.g., Eppinger and Kray, 2011). In this study we examined whether older adults benefit from salient feedback information during learning. We used an electrophysiological approach and investigated 15 younger and 15 older adults with a RL task in which they had to learn stimulusresponse associations under two learning conditions. In the positive learning conditions, participants could gain 50 Cents for a correct response but did not gain or lose money $\left(^{*} 00\right.$ Cent) for an incorrect response. In negative learning conditions, they could lose 50 Cents for an incorrect response but did not gain or lose money ( ${ }^{*} 00$ Cent) for a correct response. As the identical outcome " 00 Cent" is either better or worse than the alternative outcome depending on the learning condition, this feedback type is ambiguous. To examine the influence of feedback salience we compared this condition with a condition in which positive and negative outcomes were color-coded and thereby clearly separable. The behavioral results indicated that younger adults reached higher accuracy levels under salient feedback conditions. Moreover, the errorrelated negativity and the feedback-related negativity for losses were larger if the good-bad dimension of feedback was salient. Hence, in younger adults salient feedback facilitates the rapid evaluation of outcomes on a good-bad dimension and by this supports learning. In contrast, for older adults we obtained neither behavioral nor electrophysiological effects of feedback salience. The older adults' performance monitoring system therefore appears less flexible in integrating additional information in this evaluation process.

\section{Keywords: reinforcement learning, aging, positive and negative learning, feedback salience, ambiguous feedback,} error-related negativity, feedback-related negativity

\section{INTRODUCTION}

Given that the proportion of older adults in most Western societies is growing over the next decades, it is important for us as well as future generations to understand how we can maintain and/or improve cognitive functioning in old age. We already know that a number of fundamental cognitive processes underlying intellectual abilities, such as working memory, speed of processing, and executive control, decline with increasing age (e.g., Bialystok and Craik, 2006). We also know that the ability to learn from feedback and to adapt behavior according to positive and negative outcomes is impaired in the elderly (e.g., Samanez-Larkin et al., 2007; Zamarian et al., 2008; Eppinger and Kray, 2011). The main goal of the present study was to investigate whether older adults' ability to learn from feedback can be enhanced by providing environmental support. In particular, we made the good-bad dimension of feedback more salient in order to help older adults to better distinguish between outcomes of their actions.

To investigate age differences in learning a number of recent studies used reinforcement learning (RL) tasks in which subjects had to infer the associations between stimuli and responses depending on positive and negative feedback (monetary gains and losses). While participants performed the task electrophysiological correlates of error and feedback processing, the error-related negativity (ERN), and feedback-related negativity (FRN), were measured (Nieuwenhuis et al., 2002; Eppinger et al., 2008; Eppinger and Kray, 2011). The ERN is a component of the event-related potential (ERP) that is elicited when participants commit erroneous responses in reaction-time tasks. The component is typically observed around 50-100 ms after an erroneous response at fronto-central electrodes (Falkenstein et al., 1990; Gehring et al., 1993). A similar component, the so-called FRN is generated by negative outcomes (e.g., monetary losses) around $230 \mathrm{~ms}$ after the stimulus onset (Miltner et al., 1997). Recent findings suggest that both components are generated in the medial prefrontal cortex (mPFC; Holroyd and Coles, 2002; Holroyd et al., 2004). The mPFC is involved in performance monitoring and has been associated with dopaminergic functioning that is known to be impaired in old age (Braver and Barch, 2002; Ridderinkhof et al., 2004; Bäckman et al., 2006). Results from previous ERP studies on RL suggest that the ERN/FRN reflect expectancy violations during learning, which might be driven by phasic dopaminergic learning signals from the midbrain (Holroyd and Coles, 2002; Nieuwenhuis et al., 2002).

A number of studies so far have found that the amplitude of the ERN/FRN is reduced in older adults, suggesting age-related impairments in performance monitoring (Falkenstein et al., 2001; Mathewson et al., 2008; Wild-Wall et al., 2009). Furthermore, Nieuwenhuis et al. (2002) found reduced ERN and FRN amplitudes as well as performance impairments during RL. They concluded 
that learning impairments as well as the reduced ERN/FRN in older age may be the result of deficient dopaminergic projections from the midbrain to the mPFC. However, it seems that age differences in RL are context-dependent. For instance, Eppinger et al. (2008) showed that age-related differences in learning were restricted to situations of reward uncertainty (probabilistic reward) whereas age differences in learning were absent when feedback information was always valid (deterministic reward; see also Pietschmann et al., 2011).

In a more recent study, Eppinger and Kray (2011) examined age differences under positive and negative learning conditions. In positive learning conditions, participants received monetary gains (50 Cents) for a correct response and no gain or loss (00 Cents) for the alternative response. In the negative learning condition, participants received a monetary loss ( -50 Cents) for an incorrect response and no gain or loss (00 Cents) for the alternative response. Thus, the feedback to the alternative response is identical, but the valence is either positive or negative depending on the learning condition. One important finding of this study was that in younger adults the ERN differentiated between the four outcomes, which was not the case for older adults. That is, the ERN was significantly larger for responses that lead to negative outcomes ( -50 Cents in the negative learning condition and 00 Cents in the positive learning condition) than for responses that lead to positive outcomes (+50 Cents in the positive learning condition and 00 Cents in the negative learning condition), suggesting that younger adults were able to form relational representations of outcomes. In older adults the ERN was reduced and similar in amplitude for all four feedback types, indicating that they had problems in representing the relative value of outcomes. Given that the ERN and FRN are thought to be generated by the overlapping brain regions, similar results can be expected for the FRN. On the basis of these findings, the main question of the present study was whether feedback salience, that is, an additional feature such as the color of the feedback would help older adults to distinguish between response alternatives and whether this shows up in better learning performance.

In a recent study, Nieuwenhuis et al. (2004) examined the effect of feedback salience on outcome processing in younger adults. They applied a gambling task in which they used color-coded outcomes to either highlight the evaluation of the feedback on a gain-loss or correct-incorrect dimension. The results indicated that the FRN was sensitive to negative outcomes (either loss or incorrect responses) depending on which dimension was made more salient by the color cue. Hence, color cues can be seen as useful information that facilitates the evaluation of actions. Moreover, the findings of this study suggest that the FRN reflects a rapid evaluation process that classifies outcomes along a good-bad dimension (Nieuwenhuis et al., 2004; Hajcak et al., 2006).

Given the learning impairments of older adults in uncertain and ambiguous environments the specific aim of our study was to assess whether salient feedback promotes error and feedback processing in older adults, and by this, facilitates learning in this age group (Eppinger et al., 2008; Eppinger and Kray, 2011). To examine this, we applied a similar learning task as in our previous study in which participants learned stimulus-response assignments depending on the feedback (cf. Eppinger and Kray, 2011). In the positive learning condition, participants either received a gain of 50 Cents or a "neutral" (00 Cent) feedback, and in the negative condition, they either received a loss of 50 Cents or a "neutral" (00 Cent) feedback. To make the good-bad dimension of the outcomes more salient, feedback to incorrect responses was displayed in red and feedback to correct responses was displayed in green. We expected that error and feedback processing is facilitated by salient feedback and that this is reflected in: (a) higher accuracy and faster learning for colorcoded than for non-color feedback; (b) larger ERNs for responses that lead to negative outcomes in both learning conditions; and (c) a larger FRN for color-coded negative feedback. Given the scarce literature on age differences in feedback processing in this type of feedback, we had no strong predictions on age differences in these effects. However, we hoped to find these effects not only in younger adults but somewhat reduced also in older adults.

\section{MATERIALS AND METHODS PARTICIPANTS}

Fifteen younger ( mean age $=22.9$ years, age range $=20-28$ years, eight females) and 15 older healthy adults (mean age $=68.3$ years, age range $=65-74$ years, seven females) participated in the study. The young participants were students from Saarland University and the older participants were recruited through a database of subjects. All subjects were paid at least $22.50 €$ for participating in a 3 -h experiment. Informed consent was obtained from all participants, using a procedure approved by the Internal Review Board of Saarland University.

Consistent with a number of previous studies (e.g., Verhaeghen and Salthouse, 1997; Li et al., 2004), younger adults performed better in a perceptual speed of processing test (Digit-Symbol Substitution test adapted from Wechsler, 1982) than older adults $(M=62, \mathrm{SD}=12.6 ; M=50, \mathrm{SD}=9.5$, respectively; $p<0.01$, $\left.\eta^{2}=0.25\right)$, but they reached a lower score in a semantic knowledge test (Spot-a-Word test, adapted from Lehrl, 1977) than the elderly $(M=30, \mathrm{SD}=2.3, M=34, \mathrm{SD}=1.2$, respectively; $p<0.001$, $\left.\eta^{2}=0.50\right)$.

\section{TASK AND STIMULI}

In our learning task, participants had to learn stimulus-response assignments on the basis of deterministic feedback. Subjects were asked to respond to the stimuli by pressing one of two response keys with their left or right index finger. Similar to a previous study (Eppinger and Kray, 2011), we investigated learning under positive and negative learning conditions. In the positive learning condition, participants were rewarded (gain of 50 Cents), if they pressed the correct response key or received a neutral outcome (no gain, no loss), if they pressed the incorrect response key. In the negative learning condition, subjects lost 50 Cents for an incorrect response or received a neutral outcome for the correct response (see Figure 1).

The influence of feedback salience was examined by comparing three conditions. The first condition was our control condition ("no-color feedback") in which stimuli were displayed in black and the 50 feedback was preceded either by a "+" sign (positive learning condition) or a "-" sign (negative learning condition). The neutral feedback (00) was preceded by an asterisk in both learning conditions. Note that in the "no-color" condition the neutral feedback is ambiguous with respect to the correctness of the response. That 


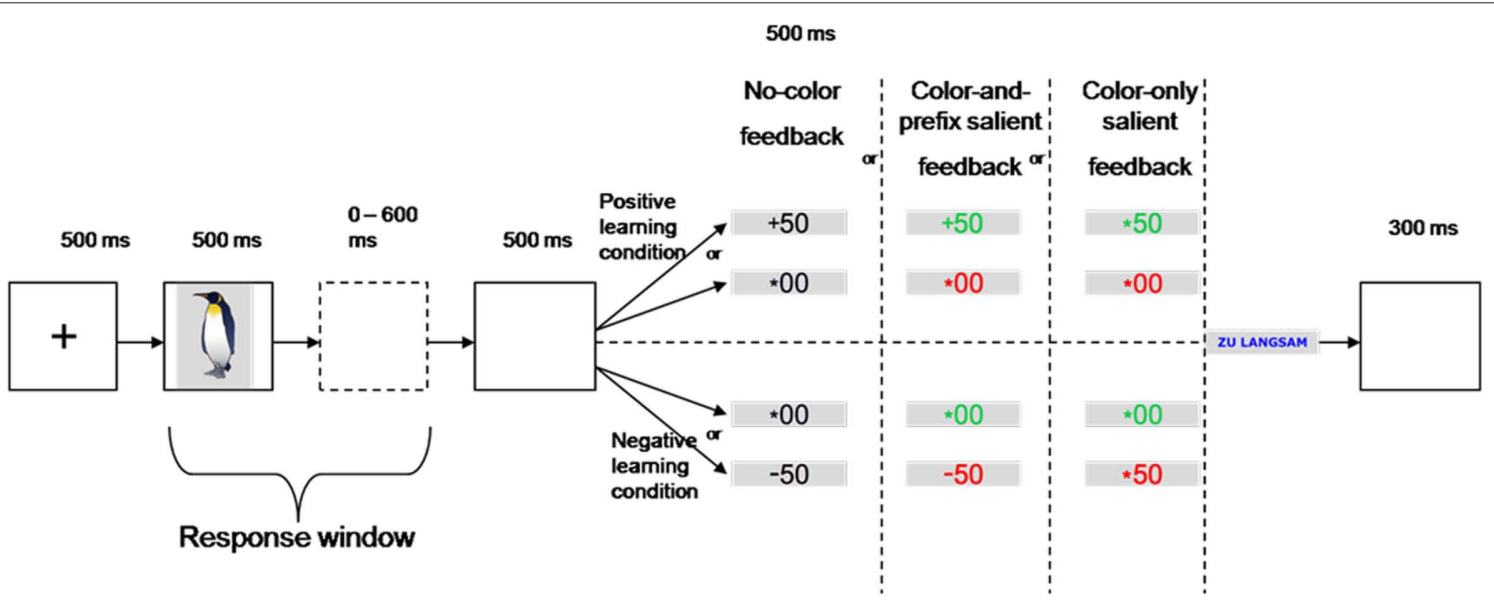

FIGURE 1 | Participants responded to an imperative stimulus picture with one of two response keys and had to learn the stimulus-response assignments by trial-and-error depending on the feedback.

is, participants have to learn whether the feedback to a response is better (negative learning condition) or worse (positive learning condition) than the feedback to the alternative response (cf. Eppinger and Kray, 2011). In the "color-and-prefix" condition we added color cues to make the good-bad dimension of the stimuli more salient (see Nieuwenhuis et al., 2004). That is, gains in the positive learning condition and neutral feedback in the negative learning condition were displayed in green. In contrast, losses in the negative learning condition and neutral feedback in the positive learning condition were displayed in red (see Figure 1). In the "color-only" condition the algebraic signs were replaced by asterisks and the valence of the outcomes was only indicated by the color. This condition served as a control condition to rule out that participants also used the prefix to learn the stimulus-response assignments. The assignment of color to learning condition was counterbalanced across subjects. The three feedback salience conditions were presented blockwise in a pre-defined sequence for all subjects.

The stimulus set consisted of 72 colored images of the Snodgrass and Vanderwart (1980) picture database. In the "no-color" condition, the feedback stimuli $(+50,-50$, and $* 00)$ were displayed in black on a gray background. In the "color-and-prefix" and the "color-only" condition, the feedback stimuli were presented in green (positive feedback) or red (negative feedback). In the "color-only" condition, the algebraic signs ("+" and "-") were replaced by an asterisk. If the response deadline was missed, the feedback stimulus "Zu Langsam" ("too slow") appeared in blue (see Figure 1).

\section{PROCEDURE}

Participants signed an informed consent and filled out a demographic questionnaire. Then, they performed the speed of processing and the semantic knowledge test. In the learning task, participants first received 90 practice trials ( 30 trials per feedback salience condition). Thereafter, they worked through 18 experimental learning blocks. Each learning block consisted of a new set of four stimuli (two per learning condition), which were presented 15 times each (60 trials per block). Each trial started with the presentation of a fixation cross that was present for $500 \mathrm{~ms}$. Then, the stimulus was presented again for $500 \mathrm{~ms}$. Response time was individually adjusted depending on the number of time-out trials to make sure that younger and older adults had similar opportunities to learn from feedback. After responding, subjects saw a blank screen for $500 \mathrm{~ms}$ and then the feedback was presented again for $500 \mathrm{~ms}$ (see Figure 1). At the end of each block, participants received feedback on their mean performance. They were motivated to maximize their gains and to minimize their losses. Furthermore, they could win a performance-dependent bonus of $€ 7.50$ in addition to the regular compensation of $€ 22.50$.

\section{DATA RECORDING AND ANALYSIS}

The stimuli were presented on a $17^{\prime}$ color monitor. The experiment was controlled by the software E-Prime 1.0 (Psychology Software Tools, Inc., Sharpsburg, PA, USA). Participants' responses and reaction times were registered on a response box (Cedrus Corporation, San Pedro, CA, USA).

EasyCaps recording caps (Easycap $\mathrm{GmbH}$ ) were used to record EEG and EOG activity from $63 \mathrm{Ag} / \mathrm{AgCL}$ electrodes (extended 10-20 system). EEG and EOG activity was recorded using Brain Amp DC Recorder and Brain Vision Recorder acquisition software (Brain Products, Germany). The left mastoid was used as reference during recording and the right mastoid was recorded as an active channel. Data were re-referenced offline to averaged mastoids. Vertical EOG was recorded from two electrodes placed below and above the right eye. Horizontal EOG was measured from electrodes attached laterally of the outer canthi of both eyes. EEG and EOG signals were filtered online from DC-70 Hz and digitized at $500 \mathrm{~Hz}$. Impedances were kept below $10 \mathrm{k} \Omega$.

Prior to analyses, the EEG data were filtered offline using a $15-\mathrm{Hz}$ low-pass filter in order to obtain more reliable peak-amplitude measures (Frank et al., 2005). Trials containing artifacts were excluded from analysis applying a threshold criterion (SD greater than $30.75 \mu \mathrm{V}$ within a sliding window of $200 \mathrm{~ms}$ ). Remaining eye-movement artifacts were corrected using a modified version of the Gratton et al. (1983) regression approach as implemented in EEProbe software (ANT Software, Enschede, Netherlands). 
For response-locked ERPs the average EEG activity -200 to $-50 \mathrm{~ms}$ pre-response served as a baseline (see Eppinger et al., 2008). To determine a peak-to-peak measure of the ERN we subtracted the most negative amplitude in a time window -50 to $150 \mathrm{~ms}$ around the response at electrode $\mathrm{FCz}$ from the preceding positive amplitude (Frank et al., 2005). Feedback-locked ERPs were baseline-corrected by subtracting the average EEG activity $-200 \mathrm{~ms}$ to feedback onset. The FRN was defined by subtracting the most negative amplitude from the preceding positive amplitude in a time window $200-400 \mathrm{~ms}$ post-feedback at FCz (see Yeung and Sanfey, 2004).

For all analyses, the Geisser-Greenhouse correction was applied where appropriate (Geisser and Greenhouse, 1958). In these cases the original $F$-values, the adjusted $p$-values, and $\varepsilon$-values are reported. Bonferroni-Holm corrections were applied when necessary $(p<0.05)$ and the corrected $p$-values are reported.

\section{RESULTS}

\section{ACCURACY DATA}

To examine age differences in learning rates, the accuracy data were averaged into five equally large bins, each bin including three trials of each of the four stimuli that were presented in every learning block (counting in a consecutive order; see Figure 2). Mean accuracy rates (in \% correct) were analyzed using an ANOVA design with the between-subjects factor Age group (young, old), the within-subjects factors Feedback salience ("no-color," "color-only," "color-and-prefix"), Learning condition (positive, negative), and Bin (bin1-bin5). The analysis revealed significant main effects of Age group, $F(1,28)=67.57, p<0.001, \eta^{2}=0.71$, Feedback salience, $F(2,56)=14.77, p<0.001, \eta^{2}=0.35$, and Bin, $F(4,112)=188.46$, $p<0.001, \eta^{2}=0.87$, as well as significant interactions between Age group and Feedback salience, $F(2,56)=6.69, p<0.002$, $\eta^{2}=0.19$, and between Age group, Feedback salience, and Bin, $F(8,224)=3.98, p<0.005, \varepsilon=0.48, \eta^{2}=0.13$. To understand the nature of this interaction we first conducted separate ANOVAs for each age group.

For the younger age group, we found main effects of Feedback salience, $F(2,28)=32.49, p<0.001, \eta^{2}=0.70$, and Bin, $F(4$, 56) $=218.96, p<0.001, \eta^{2}=0.94$, as well as an interaction between Feedback salience and Bin, $F(8,112)=5.39, p<0.003, \varepsilon=0.39$,

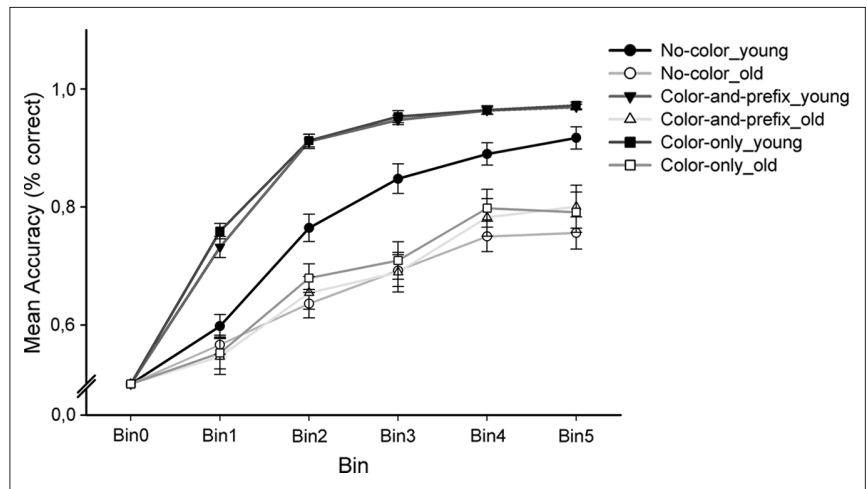

FIGURE 2 | Degrees of learning (in \% correct with SE), separately for age group and feedback salience condition. Bin 0 stands for a $50 \%$ baseline before each learning sequence. To show the course of learning, we divided learning blocks into five bins (12 trials per bin). $\eta^{2}=0.28$, indicating that learning rates were significantly different across the feedback salience conditions. Contrasts comparing the "no-color" with the two salient conditions showed that learning was better with salient feedback than with the no-color control condition, $F(1,14)=37.98, p<0.001, \eta^{2}=0.73$, but no reliable differences were found between the two salient conditions $(p=0.38)$. Separate analyses for each bin indicated that younger adults reached higher accuracy levels under salient color feedback conditions in all learning phases $\left(p s<0.02, \eta^{2} s>0.34\right)$, suggesting that they benefited from the salient feedback already at the beginning of learning (see Figure 2). For the older age group, we only found a significant main effect of Bin, $F(4,56)=57.14, p<0.001, \eta^{2}=0.80$, suggesting that the accuracy increased throughout learning. In contrast to the younger adults, we obtained no interaction with Feedback salience $(p=0.35)$, suggesting that older adults made no use of the additional color coding.

Furthermore, separate ANOVAs for each feedback salience condition showed significant main effects of Age group ( $p s<0.001$, $\left.\eta^{2}>0.45\right)$, $\operatorname{Bin}\left(p s<0.001, \eta^{2}>0.75\right)$, as well as an interaction between Age group and Bin $\left(p s<0.036, \eta^{2}>0.09\right)$, indicating faster learning in younger than in older adults under all three feedback salience conditions.

\section{ELECTROPHYSIOLOGICAL DATA}

For the analyses of the electrophysiological data, we averaged across the factor Bin because the younger participants did not commit enough error trials to analyze error-related ERPs in the salient feedback conditions.

\section{Error-related negativity}

To investigate the influence of feedback salience on error processing, the peak-to-peak measures of the ERN were analyzed using an ANOVA with the factors Age group (young, old) and Feedback salience ("no-color," "color-only," "color-and-prefix"). The ANOVA showed a significant main effect of Age group, $F(1,28)=29.96$, $p<0.001, \eta^{2}=0.52$, indicating a larger ERN for younger than older adults (see Figure 3). Furthermore, we obtained a significant main effect of Feedback salience, $F(2,56)=3.20, p<0.05, \eta^{2}=0.10$ and an interaction between Age group and Feedback salience, $F(2$, $56)=6.62, p<0.003, \eta^{2}=0.19$. Separate analyses for the two age groups revealed a larger ERN for the salient feedback conditions than for the "no-color" condition only for the younger age group $\left(p<0.002, \eta^{2}=0.49\right)$, but not for the elderly $(p=0.16$; see Figure 3$)$.

\section{Feedback-related negativity}

To examine whether feedback salience influenced feedback processing under positive and negative learning conditions, we additionally included the factor Feedback type in the ANOVA design. Hence, the peak-to-peak measures of the FRN were analyzed using an ANOVA with the factors Age group (young, old), Feedback type $(-50,-00,+50,+00)$, and Feedback salience ("no-color," "coloronly," "color-and-prefix").

The analyses revealed significant main effects of Age group, $F(1$, $28)=5.20, p<0.03, \eta^{2}=0.16$, Feedback type, $F(3,84)=4.23$, $p<0.008, \eta^{2}=0.13$, as well as significant interactions between Age group and Feedback type, $F(3,84)=4.59, p<0.005, \eta^{2}=0.14$, and between Age group, Feedback type, and Feedback salience, 

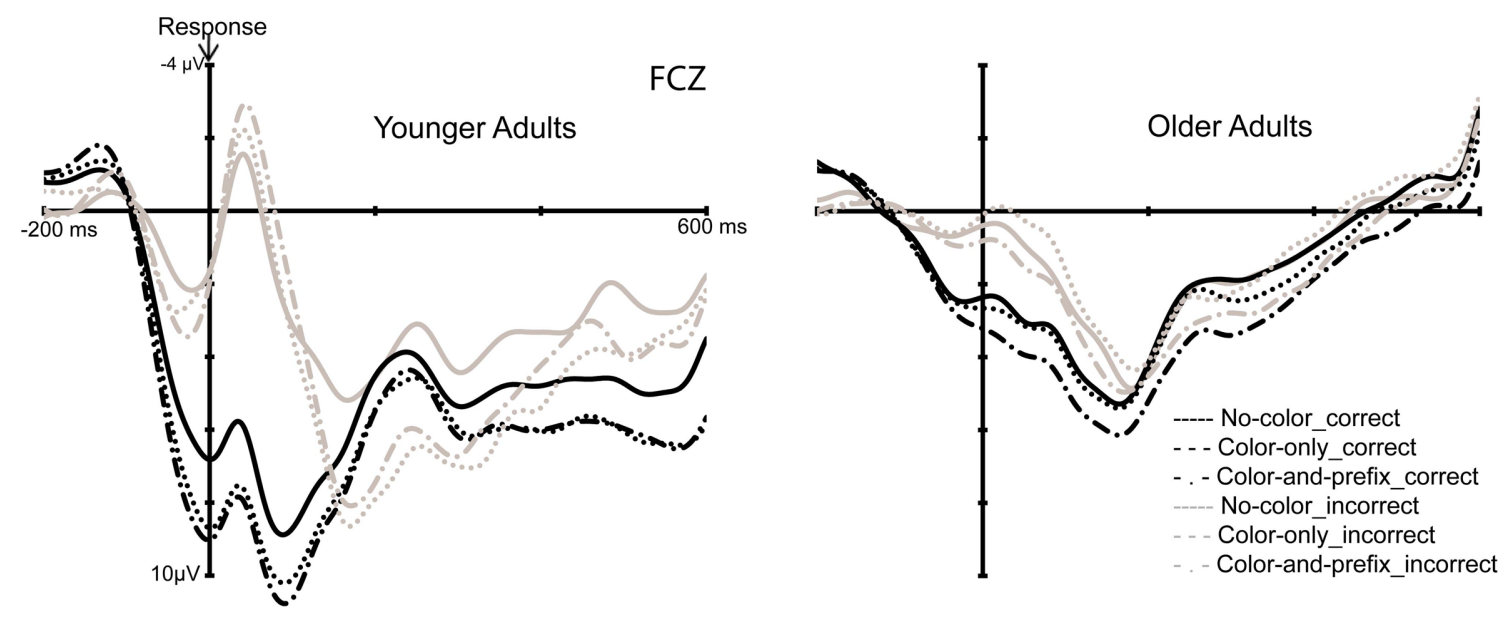

FIGURE 3 | Response-locked ERPs for the young (left) and old (right) age group.

$F(6,168)=2.46, p<0.02, \varepsilon=0.72, \eta^{2}=0.10$. Therefore, separate analyses for the factor feedback type were conducted. The results revealed a significant interaction between age group and feedback salience only for loss trials in the negative learning condition $(-50$ Feedback), $F(2,56)=3.44, p<0.04, \eta^{2}=0.11$ (see Figure 4). Further analyses showed that the FRN was larger for the two salient conditions than the "no-color" condition only for the younger age group $\left(p<0.003, \eta^{2}=0.48\right)$, but not for older age group $(p=0.91)$. That is, younger adults showed a larger FRN on loss trials in the salient than in the "no-color" condition (see Figure 4).

To further examine possible significant effects that are reflected in the three-way interaction we performed separate analyses for the two age groups and the factor feedback salience. This analysis revealed a significantly larger FRN for feedback to incorrect trials $(-50$ and -00$)$ than for feedback to correct trials $(+50$ and +00$)$ for the two feedback salience conditions in younger adults $(p s<0.04$, $\left.\eta^{2} s>0.28\right)$. No such effect was obtained for the neutral feedback condition $(p=0.52)$. Furthermore, no significant effects were obtained for older adults (all $p s>0.07$ ). Hence, the color manipulation made the Good-Bad (Correct-Incorrect) dimension of the feedback more salient for younger adults but not for older adults. A similar analysis comparing learning from positive feedback $(+50$, $-00)$ and learning from negative feedback $(-50,+00)$ did not reveal significant effects (all $p s>0.17$ ).

\section{Control analyses}

P300. Based on the results shown in Figure 4, it could be argued that the reduced FRN for loss trials ( -50 feedback) in the no-color condition is due to an increased P300 effect in this condition. Therefore, we analyzed P300 mean amplitudes at Pz with the same ANOVA design as for the FRN. The results revealed a marginally significant main effect of Feedback salience $(p=0.08)$, and a marginally significant interaction between Age group and Feedback salience $(p=0.09)$. However, in contrast to the FRN analysis, no reliable interaction between Age group, Feedback salience, and Feedback type $(p=0.44)$ was obtained. Separate analyses for the two age groups and each feedback type condition showed no significant effect of feedback salience on loss trials ( -50 feedback) for younger adults. Hence, it seems unlikely that that the reduced FRN in the neutral feedback condition in younger adults is due to an increased $\mathrm{P} 300$. For older adults the P300 was more positive going for salient feedback than for the no-color condition $(p=0.03)$ but here we obtained no salience effect for the FRN.

N1/P1. To examine whether older adults at least pay attention to feedback stimuli, we additionally investigated the early ERP components, the N1/P1 ERP complex. An analysis for the P1 mean amplitudes (at PO8, time window 72-124 ms post-feedback onset) revealed neither reliable main nor interaction effects (all ps $>0.38$ ). Even though previous findings (Hillyard and Anllo-Vento, 1998; Yordanova et al., 2004) point to an increased P1 for older adults, it should be noted that the present study was not designed to investigate these early visual components. Therefore, the results of the P1-N1 components should be taken with caution.

For the analysis of the $\mathrm{N} 1$ mean amplitudes (at PO8, time window 124-174 ms post-feedback onset) we obtained main effects of Age group, $F(1,28)=12.31, p<0.002, \eta^{2}=0.31$, and Feedback type $F(3$, $84)=45.58, p<0.001, \varepsilon=0.63, \eta^{2}=0.51$, as well as interactions between Age group and Feedback type, $F(3,84)=45.58, p<0.001$, $\varepsilon=0.63, \eta^{2}=0.51$, and between Age group, Feedback type, and Feedback salience, $F(3,84)=3.31, p<0.04, \varepsilon=0.75, \eta^{2}=0.10$. The main effect of age reflects an increased N1 component for older participants. Regarding the two-way interaction between age group and feedback type, post hoc contrasts revealed a significantly greater N1 for feedback to incorrect compared to feedback to correct responses in younger than older adults. Additionally, both age groups showed a larger $\mathrm{N} 1$ to outcomes indicating gains or losses $(+50,-50)$ compared to neutral outcomes. For younger adults, but not for the elderly, the interaction between feedback type and feedback salience was significant $\left(p<0.05, \eta^{2}=0.19\right)$. Separate analyses for the factor feedback type showed no effect of feedback salience for feedback indicating incorrect responses (i.e., $-50,-00 ; p s>0.29$ ), but an effect of feedback salience for correct feedback types (i.e., $\left.+50, p<0.003, \eta^{2}=0.48 ;+00, p<0.06, \eta^{2}=0.24\right)$. That is, younger adults showed more negative N1 components for the no-color correct feedback types than for the salient correct feedback types. 


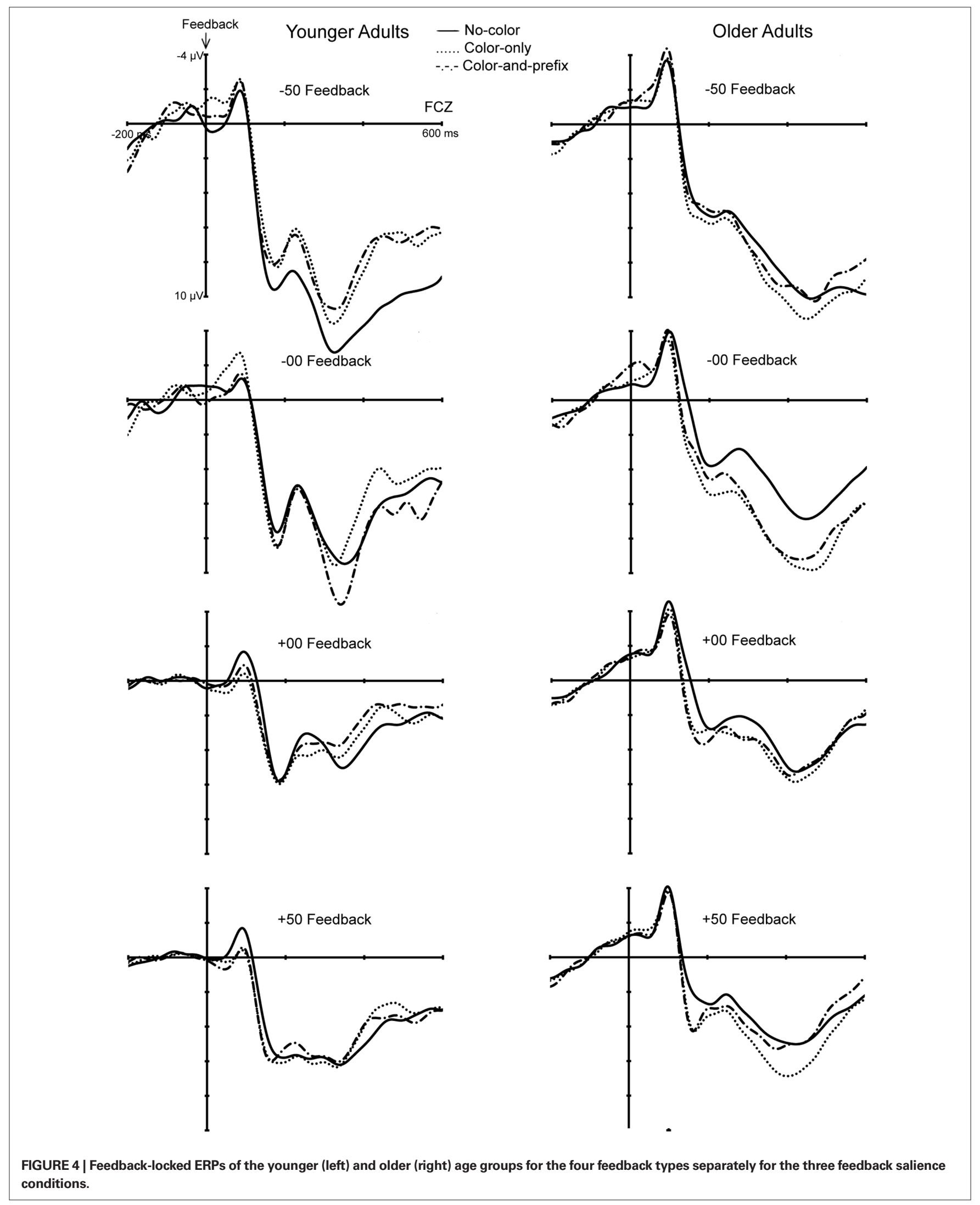


In sum, these results suggest (a) increased attention in older adults, which speaks against the hypothesis that the lower level of performance of older participants is due to a lack of attention; (b) a greater attentional focus on the behaviorally relevant negative outcomes in younger than older adults; and (c) that only younger adults seem to differentiate between salient and neutral feedback, which is reflected in an increased N1 component for no-color correct feedback compared to salient correct feedback.

\section{DISCUSSION}

The main goals of the this study were to examine (1) whether more salient feedback facilitates learning and whether this is reflected in the event-related potential correlates of error and feedback processing, and (2) whether especially older adults, who have problems in differentiating ambiguous feedback information, would benefit from more salient feedback. Similar to a previous study, we applied a reward-based learning task in which participants had to build up stimulus-response associations in two learning conditions (cf. Eppinger and Kray, 2011). In the positive learning condition, one response leads to a monetary gain and the other response leads to a neutral outcome. Hence, participants should learn to choose the responses that lead to reward. In the negative learning condition, one response leads to a monetary loss whereas the other response leads to a neutral outcome. Thus, participants should learn to avoid responses that lead to losses. Results of the previous study revealed that older, but not younger, adults had deficits in building up relational representations of outcomes, that is, they had problems to disambiguate the neutral outcomes as positive or negative. The idea of the present study was therefore to facilitate error and feedback processing by presenting more salient, i.e., color-coded, feedback. In the salient feedback conditions, the colors, red and green, unambiguously indicate the good-bad dimension of the outcome as well as the correctness of the response (see Nieuwenhuis et al., 2004). We expected that salient feedback information would help older adults to disambiguate the neutral outcomes as "good" or "bad," and that this would facilitate learning. Furthermore, we predicted that, consistent with previous findings, the salience of the outcomes should be reflected in the ERP correlates of error and outcome processing (Holroyd et al., 2004; Nieuwenhuis et al., 2004).

The behavioral data show significant age differences in the ability to use the color information for learning. The accuracy increased with learning for the group of older adults but to the same extent across all three feedback conditions, that is, older adults learned, but made no use of the additional color information. In contrast, for the younger age group the accuracy increase with learning was greater for the two salient feedback conditions than for the control condition. Moreover, the behavioral benefits due to the salient feedback occurred very early during learning indicating that younger adults were able to use the color information to disambiguate the outcomes. However, there was no additional benefit of the prefix information, suggesting that the color information per se was sufficient to produce the difference in learning. Furthermore, consistent with other studies, younger adults reached higher accuracy levels as well as a larger increase in accuracy across learning, suggesting age-related deficits in reward learning when feedback is uncertain or partially ambiguous (Hämmerer et al., 2010; Eppinger and Kray, 2011; Pietschmann et al., 2011).
Importantly, age differences in using salient feedback for learning were reflected in the electrophysiological correlates of error and feedback processing (ERN and FRN). In line with the behavioral results, we found that younger adults showed larger ERN amplitudes for the two salient feedback conditions than for the no-color control condition. No amplitude differences between the "color-only" and "color-and-prefix" condition were observed. These results support the idea that younger adults can use the color coding for a better representation of the correct response, which results in a larger mismatch, if they commit an error (Holroyd and Coles, 2002; Eppinger et al., 2008). Consistent with their performance impairments the ERN was largely reduced for the older adults as compared to the younger adults (Eppinger et al., 2008; Hämmerer et al., 2010; Pietschmann et al., 2011). Furthermore, the ERN was not modulated by the different feedback salience conditions, which is also in line with the behavioral data. Hence, the color coding was obviously not the appropriate context information to facilitate learning in older adults.

Consistent with our predictions and with previous data, we found a larger FRN for the salient than the "no-color" condition for younger adults (Nieuwenhuis et al., 2004) but not for older adults. Interestingly, the salience effect in younger adults was only observed for loss outcomes ( -50 Feedback) but not for neutral outcomes. Based on previous findings, we expected that the color coding of feedback stimuli would be primarily useful for disambiguating the neutral outcomes on a good-bad dimension (cf. Nieuwenhuis et al., 2004). One explanation for this finding could be that the outcomes are not processed relative to each other within a certain condition but rather in context of all possible outcomes in the experiment (Holroyd et al., 2004). Such an argumentation would suggest that although the neutral outcomes in the positive learning condition may acquire some negative "valence" with learning, they are still perceived as less aversive than outcomes indicating a loss. The present findings suggest that feedback processing as indexed by the amplitude of the FRN is associated with the evaluation of outcomes along a relative good-bad dimension, which depends on the range of all possible outcomes in the experiment.

In contrast to the younger adults, we did not obtain a significant effect of reward salience on learning in older adults. The absence of a behavioral learning benefit for salient feedback is paralleled by the absence of a modulation in the ERN and FRN components by reward salience. Our results concerning age differences in reward learning are quite consistent with previous findings: (a) although older adults are able to differentiate between correct and incorrect responses, this process is less efficient than in younger adults, as indicated by a reduced ERN amplitude in the elderly (Falkenstein et al., 2001; Nieuwenhuis et al., 2002; Eppinger et al., 2008), when the learning context includes ambiguous or overlapping information; and (b) the rapid evaluation of feedback is impaired in older adults, as indicated by a reduced FRN amplitude (Eppinger et al., 2008; Wild-Wall et al., 2009; Hämmerer et al., 2010).

Our hope was that the additional color coding would help older adults to better distinguish between positive and negative outcomes and that this would promote learning. The data of the present study clearly show that older adults made no use of salient feedback. The reasons for this are unclear. One explanation for these results could be that due to general slowing in speed of processing of older adults 
(e.g., Salthouse, 1996; Li et al., 2001), they are less able to process the color information in addition to the feedback value and to relate the reward representation to the previous stimulus and the corresponding response. Note that the time window between presentation of the feedback stimulus and the onset of the next trial was only $300 \mathrm{~ms}$. Thus, if one would provide more time for context updating (relate the outcome to the stimulus-response mapping) after feedback presentation, older adults may be able to make use of the salient information. Consistent with this view one could argue that the presentation time of the feedback (500 ms, see Figure 1) was too short for the older adults to adequately process the outcomes and to relate them to their responses. Based on this assumption one would predict that elderly should also pay less attention to the outcomes as reflected in a reduced $\mathrm{N} 1$ component to the feedback. To address this issue we performed a similar analysis for the $\mathrm{N} 1$ as we did for the FRN. The results show a larger N1 for older compared to younger adults, suggesting that, if at all, the older adults paid more attention to the feedback than the younger adults (see also Hillyard and Anllo-Vento, 1998; Yordanova et al., 2004). Interestingly, the results of this analysis also indicate that younger and older adults differ with respect to which feedback type they pay most attention to. Whereas younger adults show greater (more negative) $\mathrm{N} 1$ components to feedback indicating errors $(-50$, $-00)$, both age groups show a greater N1 to outcomes indicating gains or losses $(+50,-50)$ compared to outcomes indicating neutral outcomes. Moreover, in younger adults the N1 is greater for feedback to correct responses $(+50,+00)$ in the more challenging neutral compared to the salient feedback condition. No such effect is obtained for older adults. Taken together, these findings suggest that reward and punishment information affect early attentional processes differentially in younger and older adults. Although it should be noted that this experiment was not designed for the analysis of early visual components we think that these are potentially important findings that should be addressed in future experiments (for similar findings in younger adults, see Hickey et al., 2010).

\section{REFERENCES}

Bäckman, L., Nyberg, L., Lindenberger, U., Li, S. C., and Farde, L. (2006). The correlative triad among aging, dopamine, and cognition. Neurosci. Biobehav. Rev. 30, 791-807.

Bialystok, E., and Craik, F. I. M. (eds). (2006). Lifespan Cognition: Mechanisms of Change. New York: Oxford University Press.

Braver, T. S., and Barch, D. M. (2002). A theory of cognitive control, aging cognition, and neuromodulation. Neurosci. Biobehav. Rev. 26, 809-817.

Eppinger, B., and Kray, J. (2011). To choose or to avoid: age differences in learning from positive and negative feedback. J. Cogn. Neurosci. 23, 41-52.

Eppinger, B., Kray, J., Mock, B., and Mecklinger, A. (2008). Better or worse than expected? Aging, learning, and the ERN. Neuropsychologia 46, 521-539.

Falkenstein,M.,Hohnsbein,J.,Hoormann, J., and Blanke, L. (1990). "Effects of errors in choice reaction tasks and
Another explanation for the lacking salience effect could be that older adults have problems in maintaining all relevant representations in working memory and to relate them to each other. Note that in order to enhance task performance subjects have to maintain the presented stimulus and the corresponding response over time and to relate these representations to the value of the feedback. Because of working-memory limitations in older adults (Hasher and Zacks, 1988; Salthouse et al., 1991), they may have problems to maintain and integrate additional information such as the color that would help them to create a simpler feedback that only differs along a good vs. bad dimension.

A third interpretation could be that older adults are less able to focus on specific aspects of the feedback during learning. Whether such age-related deficits in the ability to attentionally focus on the relevant stimulus dimension are the result of reduced processing capacities or whether these effects reflect a more general problem in the allocation of attentional control is an open question. Therefore, further studies are needed to clarify the lack of using salient feedback in older adults.

To summarize and conclude, younger adults showed better task performance and faster learning when the feedback contained an additional feature that allowed a rapid evaluation of outcomes as good or bad. Behavioral learning benefits are fully consistent with modulations of the ERN and FRN. The ERN amplitude was larger for salient feedback and the FRN was larger for the negative-associated feedback as for the gain feedback only in younger adults. Hence, in contrast to older adults, performance monitoring in younger adults is flexible in integrating new information for a better adaptation to the learning context.

\section{ACKNOWLEDGMENTS}

This work was funded by the German Research Foundation (Deutsche Forschungsgemeinschaft; grant SFB 378 for project EM2). We thank our participants for their contribution to the study.

Hajcak, G., Moser, J. S., Holroyd, C. B. and Simons, R. F. (2006). The feedback-related negativity reflects the binary evaluation of good versus bad outcomes. Biol. Psychol. 71, 148-154.

Hämmerer, D., Li, S.-C., Müller, V., and Lindenberger, U. (2010). Lifespan differences in electrophysiological correlates of monitoring gains and losses during probabilistic reinforcement learning. J. Cogn. Neurosci. 23, 579-592.

Hasher, L., and Zacks, R. T. (1988). "Working memory, comprehension, and aging," in The Psychology of Learning and Motivation, Vol. 22 ed. G. H. Bower (San Diego, CA: Academic Press), 193-225.

Hickey, C., Chelazzi, L., and Theeuwes, J. (2010). Reward changes salience in human vision via the anterior cingulate. J. Neurosci. 18, 11096-11103.

Hillyard, S.A., and Anllo-Vento, L. (1998). Event-related brain potentials in the study of visual selective attention. Proc. Natl. Acad. Sci. 95, 781-787.
Holroyd, C. B., and Coles, M. G.H. (2002) The neural basis of human error processing: reinforcement learning, dopamine, and the error-related negativity. Psychol. Rev. 109, 679-709.

Holroyd, C. B., Larsen, J. T., and Cohen, J. D. (2004). Context dependence of the event-related potential associated with reward and punishment. Psychophysiology 41, 245-253.

Lehrl, S. (1977). MehrfachwahlWortschatz-Test $B \quad(M W T-B)$. [Multiple-Choice Knowledge Test-B (MWT-B)]. Erlangen: Straube.

Li, S. C., Lindenberger, U., Hommel, B. Aschersleben, G., Prinz, W., and Baltes, P.B. (2004). Transformations in the couplings among intellectual abilities and constituent cognitive processes across the lifespan. Psychol. Sci. 15, 155-163.

Li, S. C., Lindenberger, U., and Sikström, S. (2001). Aging cognition: from neuromodulation to representation. Trends Cogn. Sci. (Regul. Ed.) 5, 479-486.

Mathewson, K. J., Dywan, J., Snyder, P. J., Tays, W. J., and Segalowitz, S. (2008). 
Aging and electrocortical response to error feedback during a spatial learning task. Psychophysiology 45, 936-948.

Miltner, W. H., Braun, C. H., and Coles, M. G. H. (1997). Event-related brain potentials following incorrect feedback in a time-estimation task: evidence for a "generic" neural system for error detection.J. Cogn. Neurosci. 9, 788-798.

Nieuwenhuis, S., Ridderinkhof, K. R., Talsma, D., Coles, M. G. H., Holroyd, C. B., Kok, A., and van der Molen, M. W. (2002). A computational account of altered error processing in older age: dopamine and the error-related negativity. Cogn. Affect. Behav. Neurosci. 2, 19-36.

Nieuwenhuis, S., Yeung, N., Holroyd, C. B., Schurger, A., and Cohen, J. D. (2004). Sensitivity of electrophysiological activity from medial frontal cortex to utilitarian and performance feedback. Cereb. Cortex 14, 741-747.

Pietschmann, M., Endrass, T., Czerwon, B., and Kathmann, N. (2011). Aging, probabilistic learning and performance monitoring. Biol. Psychol. $86,74-82$.
Ridderinkhof, K. R., Ullsperger, M., Crone, E. A., and Nieuwenhuis, S. (2004). The role of the medial frontal cortex in cognitive control. Science 306, 443-447.

Salthouse, T. A. (1996). The processingspeed theory of adult age differences in cognition. Psychol. Rev. 103, 403-428.

Salthouse, T. A., Babcock, R. L., and Shaw, R. J. (1991). Effects of adult age on structural and operational capacities in working memory. Psychol. Aging 6, 118-127.

Samanez-Larkin, G. R., Gibbs, S. E. B., Khanna, K., Nielsen, L., Carstensen, L. L., and Knutson, B. (2007). Anticipation of monetary gain but not loss in healthy older adults. Nat. Neurosci. 10, 787-791.

Snodgrass, J. G., and Vanderwart, M. (1980). A standardized set of 260 pictures: norms for name agreement, image agreement, familiarity, and visual complexity. J. Exp. Psychol. Hum. Learn. 6, 174-215.

Verhaeghen, P., and Salthouse, T.A. (1997). Meta-analyses of age-cognition relations in adulthood: estimates of linear and nonlinear age effects and structural models. Psychol. Bull. 122, 231-249.

Wechsler, W. (1982). Handanweisung zum Hamburg-Wechsler-Intelligenztest für Erwachsene (HAWIE). [Manual for the Hamburg-Wechsler Intelligence Test for Adults]. Bern: Huber.

Wild-Wall, N., Willemsen, R., and Falkenstein, M. (2009). Feedbackrelated processes during a time production task in young and older adults. Clin. Neurophysiol. 120, 407-413.

Yeung, N., and Sanfey, A. G. (2004) Independent coding of reward magnitude and valence in the human brain. J. Neurosci. 24, 6258-6264.

Yordanova, J., Kolev, V., Hohnsbein, J., and Falkenstein, M. (2004). Sensorimotor slowing with ageing is mediated by a functional dysregulation of motorgeneration processes: evidence from high-resolution event-related potentials. Brain 127, 351-362.

Zamarian, L., Sinz, H., Bonatti, E. Gamboz, N., and Delazer, M. (2008). Normal aging affects decisions under ambiguity, but not decisions under risk. Neuropsychology 22, 645-657.

Conflict of Interest Statement: The authors declare that the research was conducted in the absence of any commercial or financial relationships that could be construed as a potential conflict of interest.

Received: 27 April 2011; paper pending published: 23 May 2011; accepted: 11 July 2011; published online: 11 August 2011. Citation: Herbert M, Eppinger B and Kray $J$ (2011) Younger but not older adults benefit from salient feedback during learning. Front. Psychology 2:171. doi: 10.3389/ fpsyg.2011.00171

This article was submitted to Frontiers in Cognition, a specialty of Frontiers in Psychology.

Copyright (C) 2011 Herbert, Eppinger and Kray. This is an open-access article subject to a non-exclusive license between the authors and Frontiers Media SA, which permits use, distribution and reproduction in other forums, provided the original authors and source are credited and other Frontiers conditions are complied with. 